Revista

\title{
Cuidando de idosos com Demência: um estudo a partir da prática ambulatorial de enfermagem
}

\author{
Caring the elderly with Dementia: a study of the ambulatorial nursing practice \\ Cuidando de ancianos con Demencia: pratica de enfermería en el ambulatorio
}

\section{Rosimeire Ferreira Santana}

Mestre em Enfermagem. Especialista em Psicoterapia. Professora da Universidade Estácio de Sá. Enfermeira do Pólo de Neurogeriatria da Prefeitura do Rio de Janeiro. rose79@uol.com.br

Iraci dos Santos

Doutora em Enfermagem. Professora Titular da FENF-UERJ

\section{Célia Pereira Caldas}

Doutora em Enfermagem. Professora Adjunto da FENF-UERJ. Vice-Diretora da UnATI-UERJ.

\section{RESUMO}

Investiga-se o cuidado realizado por cuidadores a idosos com demência, tendo como objetivos: Descrever as características de dezesseis idosos e seus cuidadores atendidos no ambulatório de neurogeriatria, do Rio de Janeiro, em 2004 ; Identificar diagnósticos de enfermagem e cuidados realizados por cuidadores, através de EDG, MEEM, AIVDs, AVDs aplicados na consulta de enfermagem. Aplicou-se o método quantitativo, descritivo, tipo estudo de caso, obtendo-se os resultados: No cliente - Desorientação, agressividade, pele seca, incontinência urinária, distúrbio no relacionamento familiar; No cuidador - Comunicação cuidador/ cliente ineficiente, desgaste físico, falta de conhecimento sobre cuidados; Cuidados realizados - alimentação, medicação e higiene. Concluise que são necessárias ações educativas de enfermagem visando à qualidade do cuidado ao idoso e seus familiares.

Descritores: Idoso; Demência; Cuidadores.

\section{ABSTRACT}

The care provided by caregivers to elderly people with dementia is investigated having as objectives: to describe the characteristics of sixteen elderly and their caregivers in attendence at the neurogeriatrical ambulatory of Rio de Janeiro during 2004; to identify nursing and care diagnosis used by caregivers, through EDG, MEEM, AIVDs and AVDs applied in nursing assessments. The quantitative descriptive method was employed, as a study of each case, obtaining the results: in the client-Disorientation, aggressiveness, skin drought, urinary incontinence, disturbance in the family relationship; In the caregivers- inefficient caregiver/ elderly communication, physical fatigue, lack of knowledge about care; accomplished types of care - feeding, medication and hygiene. In conclusion, educational nursing actions are necessary to improve the care quality to the elderly and their family. Descriptors:Aged; Gerontology; Dementia; Caregivers.

\section{RESUMEN}

Fue investigado la calidad del cuidado ejecutado por los cuidadores a los ancianos con demencia, teniendo como objetivos: - Describir el perfil de 16 ancianos y sus cuidadores acojidos en el ambulatorio de la neurogeriatria, del Rio de Janeiro, en el año 2004; Identificar diagnósticos de enfermería y cuidados ejecutados por los cuidadores, través de EDG, MEEM, AIVDs, AVDs que tuve aplicación en la consulta de enfermería. Fue aplicado el método de investigación de estudio de caso, obteniéndose los resultados: En el enfermo - Desorientación, agresividad, píele seca, disturbio urinario, disturbio en el reracionamiento familiar; En el cuidador - Comunicación cuidador/ enfermo ineficaz, desgaste, falta de conocimiento sobre cuidados; Cuidados ejecutados - alimentación, medicación y higiene. Se concluyó que son necesarias acciones educativas de enfermería con el objetivo de calificar el cuidado al anciano y sus familiares.

Descriptores: Anciano; Demencia, Cuidadores.

Santana RF, Santos I, Caldas CP. Cuidando de idosos com Demência: um estudo a partir da prática ambulatorial de enfermagem. Rev Bras Enferm 2005 jan-fev; 58(1):44-8.

\section{INTRODUÇÃO}

Na última década as enfermeiras da área de saúde do idoso se viram envolvidas e desafiadas, tanto pela equipe multiprofissional, como pelos familiares no atendimento especializado de enfermagem ao idoso com demência e ao suporte familiar/ cuidador. Isto não se deve somente ao aumento do número de casos de demência, mas especialmente pelo compromisso da enfermagem no cuidado humano.

A enfermeira é a profissional mais requisitada nos 10 a 15 anos de progressão da doença, orientando a adaptação dos cuidados a progressiva dependência do idoso; a instrumentalização do familiar para o cuidado; e estimulando o autocuidado e a preservação da auto-estima no binômio idoso-família. Portanto se impõe a necessidade de cuidados de enfermagem sistematizados, dando prioridade a aqueles relacionados às atividades de vida diária e à prevenção de incapacidades e complicações, juntamente com a educação dos familiares.

A ocorrência das demências aumenta exponencialmente com a idade, dobrando, 
aproximadamente, a cada 5,1 anos, a partir dos 60 anos de idade. Após os 64 anos de idade a prevalência é de cerca de 5 a $10 \%$, após os 75 anos de idade de 15 a $20 \%$, entre os 85 anos $32 \%$, podendo atingir $38,6 \%$ dentre aqueles com 90 e 95 anos. Fator preocupante já que a população "mais idosa" vem aumentando concomitante com 0 envelhecimento populacional(1-3).

Temos com problemas nesta investigação: Qual é a qualidade do cuidado domiciliar desenvolvido por cuidadores ao cliente idoso com demência? Quais as características dos idosos com demência atendidos no ambulatório de neurogeriatria? Quais são as características de seus cuidadores?

Justifica-se a sua importância pela necessidade de prevenir dificuldades de ordem física, sociais, emocionais e financeiras, para o idoso e sua família. Sendo assim, temos como objetivos: descrever características de 16 idosos atendidos no ambulatório de neurogeriatria; descrever características dos cuidadores destes idosos; Identificar os problemas, diagnósticos de enfermagem e cuidados por cuidadores através da consulta de Enfermagem.

\section{MÉTODO}

Aplicou-se o método quantitativo, descritivo, caracterizado em estudo de caso. Para tanto realizou-se consultas de enfermagem para 16 idosos com demência que foram acompanhados por seus cuidadores. Durante a consulta foram aplicados os instrumentos de coleta de dados (ICD) compostos pelas variáveis: 1) características sóciodemográficas (faixa etária, grau de instrução, renda familiar) dos idosos; 2) características sociodemográficas dos cuidadores; 3) Referentes às práticas de enfermagem desenvolvidas pelos cuidadores.

Para coletar os dados sobre os problemas apresentados pelos idosos com demência realizou-se uma revisão dos prontuários, consultandose o impresso próprio da Consulta de Enfermagem Neurogeriátrica (CEN) e os resultados dos seguintes ICDs, aplicados nesta pesquisa, caracterizando-se a avaliação profissional de enfermagem ao cliente idoso com demência: a Escala de Depressão Geriátrica (EDG), MiniExame do Estado Mental (MEEM), Escala de Atividades Instrumentais de Vida Diária (AIVDs) Lawton, e Escala de Atividades de Vida Diária (AVDs) Katz, instrumentos amplamente conhecidos e utilizados na saúde do idoso, que compõem o prontuário.

O campo da pesquisa foi o Núcleo de Atenção ao Idoso (NAI), da Universidade Aberta para Terceira Idade (UnATI) da Universidade do Estado do Rio de Janeiro (UERJ), onde se encontram cadastrados e são atendidos os 16 clientes sujeitos desta investigação. Nesta pesquisa caracterizam-se os grupos de idosos, de cuidadores e a distribuição dos indivíduos em relação aos problemas identificados nas consultas de enfermagem. Os resultados são apresentados através das freqüências em números absolutos (Fi). Registra-se que devido ao estudo de caso se restringir a uma pequena amostra (16 clientes), não foi verificada a freqüência percentual.

O estudo foi desenvolvido de modo a garantir o cumprimento dos preceitos estabelecidos na Resolução 196/96 da Comissão Nacional de Ética em Pesquisa do Ministério da Saúde, tendo sido aprovado pelo Comitê de Ética da Universidade Aberta da Terceira Idade - UERJ.

\section{RESULTADOS}

\subsection{Características dos idosos}

Os resultados são descritos a partir dos dados obtidos em quatro Consultas de Enfermagem em Neurogeriatria realizadas com os 16 sujeitos da pesquisa. Quanto às variáveis faixa etária e sexo observase que, os clientes situam-se na faixa de 60 a 99 anos, predominando sete pessoas na faixa de 70 a 75 , que se distribuem em duas do sexo masculino e cinco, do feminino. Constatou-se a predominância de 11 mulheres e apenas um cliente acima de 90 e do sexo masculino.

Quanto ao grau de instrução, os clientes distribuem-se em todos os graus, predominando nove com o Ensino Fundamental Completo. Apenas um cliente situa-se respectivamente nos graus de Analfabeto e Ensino Superior Completo. Na distribuição da renda salarial e procedência desta observou-se que, todos os idosos possuem renda cujo valor varia de um a quinze salários mínimos, sendo procedente de: aposentadoria, pensão, aposentadoria e pensão e ajuda de outros. Predominam oito clientes que recebem apenas um salário mínimo que, na época do estudo era equivalente a $R \$ 240,00$. Ressalte-se que 0 único que recebe ajuda, se refere ao valor citado. Apenas três idosos recebem oito salários mínimos, enquanto um sujeito recebe pensão no valor de $\mathrm{R} \$ 3.600,00$

Os sujeitos do estudo moram predominantemente (10) em sua própria casa, enquanto cinco residem com um familiar. Apenas um cliente mora em Casa de Repouso. Os núcleos familiares investigados incluem: Filha, filho, esposa, prima e irmão. Foram encontrados três idosos que moram sozinhos. Entre estes há um homem que é cuidado pela filha e duas mulheres, cuidadas pela vizinha e por um sobrinho, respectivamente.

\subsection{Características dos Cuidadores}

Mendes $^{(4)}$ define cuidador distinguindo 0 cuidador principal e 0 secundário. 0 cuidador principal é aquele que tem a total ou a maior responsabilidade pelos cuidados prestados ao idoso dependente, no domicílio. Os cuidadores secundários seriam os familiares, voluntários e profissionais, que prestam atividades complementares. Usa-se a denominação de cuidador formal (principal ou secundário) para o profissional contratado (atendente de enfermagem, acompanhante, empregada doméstica, etc) e de cuidador informal, usualmente, os familiares, amigos e voluntários da comunidade.

Foram identificados 23 cuidadores - 13 principais e 10 secundários (Alguns idosos possuem mais de um cuidador). Cinco cursaram o ensino superior completo, quatro possuem ensino médio completo, cinco possuem ensino médio incompleto, dois possuem fundamental incompleto e quatro, ensino fundamental incompleto. Apenas dois cuidadores são do sexo masculino. Quanto à idade, seis encontram-se na faixa de 60 a 70 anos, uma cuidadora com 78 anos e os demais entre 36 e 59 anos.

Quanto ao grau de parentesco destes cuidadores, foram encontradas 10 filhas e três esposas. Outros parentescos são: sobrinho, irmão, filho. Todos são cuidadores principais. Dentre os cuidadores secundários temos como grau de parentesco: prima e neta, e os demais não possui grau de parentesco, temos: vizinha e acompanhante.

\subsection{Resultado dos Testes - MEEM, EDG, LAWTON E KATZ}

Apesar da grande critica aos testes por não levarem em conta a subjetividade dos indivíduos, na prática, os testes são uma forma de se identificar rapidamente os problemas do idoso. Este chega quase sempre trazido por um familiar que está com dificuldade em lidar com as questões de cuidado e precisa de uma intervenção profissional imediata. Se os testes forem utilizados junto com a pessoa idosa e seu cuidador, num processo dialógico, sem agredir ou expor o idoso à suas dificuldades, são um ótimo meio de avaliação rápida que expressa de forma objetiva a cognição, humor e o desempenho nas atividades diárias.

Quando não se utilizam os testes, existe a possibilidade dos cuidadores apontarem que na consulta o idoso apresentou um desempenho melhor do que em casa, causando uma frustração no cuidador. Hoje percebemos que os testes podem facilitar a comunicação entre o idoso, família e profissional, auxiliando a identificação dos problemas. Tanto, que neste serviço, a avaliação é realizada a cada três meses com ótima receptividade e adesão dos cuidadores.

A EDG (Escala de Depressão Geriátrica) pode auxiliar a identificar casos sugestivos de depressão. É freqüente a confusão entre quadros de demência e depressão. 0 idoso demenciado dificilmente relata alguma dificuldade de memória e de alteração do humor, e nas consultas eles geralmente utilizam a racionalização (meio utilizado pelos idosos com demência para explicar algo que ele não consegue responder) para não demonstrar suas falhas de memória.

Ao contrário do idoso demenciado, o idoso deprimido geralmente apresenta queixas de esquecimento. Ao aplicarmos a EDG é possível identificar a dificuldade na compreensão das perguntas e o processo de racionalização no idoso com demência que tenta explicar cada questão respondida. O idoso deprimido geralmente responde "não sei", e não se esforça para buscar a resposta nem explica o porque desta 
resposta. Na EDG, resultados entre 5 a 7 devem ser investigados e acima de 7 indicam provável depressão.

O MEEM (Mini Exame do Estado Mental) é considerado na atualidade, internacionalmente, método padrão de triagem diagnóstica de demências ${ }^{(3)}$. Quanto à avaliação dos seus resultados, a pontuação do MEEM é relacionada ao nível de escolaridade. Este teste foi validado no Brasil (2) com os seguintes pontos de corte: 21 para nível superior, 18 para cinco anos ou mais de escolaridade, e 14 para analfabeto, qualquer valor abaixo de 13 evidenciaria a necessidade de investigação para síndrome demencial.

Para se avaliar o grau de dependência utiliza-se o método de avaliação funcional. Esse método, bastante conhecido e aplicado na prática geriátrica, tem se mostrado um indicador sensível e relevante para avaliar necessidades e determinar a utilização de recursos ${ }^{(5)}$.

A "função" é definida como a capacidade de um indivíduo se adaptar aos problemas cotidianos,ou seja, aquelas atividades que the são requeridas por seu entorno imediato. A função é avaliada com base na capacidade de execução das atividades da vida diária (AVDs).

As AVDs, por sua vez se dividem em: (a) atividades básicas da vida diária - tarefas próprias do autocuidado, como alimentar-se, vestir-se, controlar os esfíncteres, banhar-se, locomoverse, etc.; (b) atividades instrumentais da vida diária - indicativas da capacidade para levar uma vida independente na comunidade, como realizar as tarefas domésticas, compras, administrar as próprias medicações, manusear dinheiro, etc. A Escala de Katz avalia as atividades básicas da vida diária. Seu resultado é dado por letras que representam o número de atividades que o indivíduo é capaz de realizar sozinho. A para nenhuma perda, $B$ para uma perda até $\mathrm{G}$ que indica perda nas seis atividades básica de vida diária indicando dependência total pelo cuidador. A escala de LAWTON avalia as atividades instrumentais da vida diária. Sua pontuação vai até 21 , que caracteriza o indivíduo independente e números abaixo deve ser relacionada à dependência.

Na população estudada todos os indivíduos têm dificuldades cognitivas com MEEM abaixo de 13, porém não apresentam características depressivas com EDG abaixo de 5, e apresenta ainda 5 indivíduos que não foram possíveis de aplicar os testes por não compreenderem as questões. Quanto à escala de atividades Instrumentais de vida diária (LAWTON) a maioria é dependente, com três indivíduos necessitando de ajuda em duas atividades e 13 dependentes de forma parcial ou total nas sete atividades, uma característica comum do idoso com síndrome demencial que primeiro perde as atividades fora de casa. E na escala de KATZ temos que cinco são totalmente independentes para os cuidados pessoais, e a grande maioria onze indivíduos necessitando de ajuda ou não realizam cuidados pessoais, o que demonstram a grande dependência destes indivíduos em relação ao cuidador, assim como a progressão da dependência em relação à evolução dos estágios da demência. No quadro 1 podemos visualizar adequadamente a distribuição do desempenho dos clientes nos quatro testes apresentados.

3.4 Cuidados prestados pelos cuidadores

$\mathrm{Na}$ análise da tabela 1 comprovamos a extrema necessidade da enfermagem orientar os cuidados. Todos os idosos precisam dos cuidadores para preparar a comida e tomar remédios, isto implica num grau de dependência importante.

Tabela 1- Distribuição dos cuidados prestados pelos cuidadores dos clientes pesquisados. Rio de Janeiro, Ambulatório de Neurogeriatria, 2004.

\begin{tabular}{cc}
\hline Cuidados que presta & $\mathrm{Fi}$ \\
\hline Preparo da alimentação & 16 \\
Administra medicamentos Banho & 16 \\
Auxilia a vestimenta & 12 \\
Auxilia na movimentação & 12 \\
Administração de alimentação & 9 \\
Faz toalete & 8 \\
Realiza curativo & 8 \\
\hline
\end{tabular}

Doze idosos são dependentes para os cuidados com higiene, esta tarefa parece fácil mais conforme estudos anteriores ${ }^{(6)} 0$ banho suscita no mínimo três domínios de habilidades e atividades do cuidador: 1sexualidade e intimidade, 2- questões relativas a dependência, e 3desgaste do cuidador. Sendo descrita por vários autores ${ }^{(6-10)}$ como a atividade mais complexa e de maior desgaste encontrada nas pesquisas com cuidadores, o banho é uma queixa constante de maus tratos e de negligência ao idoso.

São nove os idosos que necessitam de ajuda para locomover-se, conforme estudos de Validação do Diagnóstico de Enfermagem Mobilidade Física Prejudicada em idosos institucionalizados ${ }^{(1)}$ a mobilidade é um dos cuidados de enfermagem que mais deve ser investido para prevenção de perdas motoras no indivíduo que envelhece, conseqüentemente promover sua independência, isso pode ser conseguido por oficinas ou ensino ao cuidador de exercícios de mobilização passiva e ativa, de força, e de equilíbrio.

A perda da movimentação causa efeitos negativos no idoso (inutilidade) e na família pela dependência e por dores principalmente nas "costas" pelo esforço físico em auxiliar na movimentação incorretamente, não utilizando princípios de mecânica corporal. Daí a importância da orientação da enfermagem, de como prestar os cuidados com maior destreza e com 0 menor gasto de "energia", uma das melhores formas de diminuir o estresse do cuidador.

O item faz o toalete encontrado em metade dos indivíduos indica que primeiro o indivíduo necessita de ajuda no banho, que é uma atividade mais complexa necessitando de maiores habilidades cognitivas, porém somente no evoluir da síndrome o individuo precisa de ajuda no uso do banheiro. A administração de alimentos é uma das últimas habilidades a ser perdida, ela indica comprometimento grave das funções cognitivas e motoras. Deve se dar bastante atenção na avaliação de como o cuidador oferece a comida, porque concomitante a perda da capacidade de se alimentar advém a disfagia, ocasionando engasgos e suas complicações de broncoaspiração, pneumonias e perda de peso. Nesse momento o cuidador necessita de atendimento em equipe interdisciplinar para dar conta das novas necessidades

\begin{tabular}{|c|cc|cc|c|c|c|}
\hline \multicolumn{3}{|c}{ MINI-MENTAL (MEEN) } & \multicolumn{2}{c|}{ Escala de Depressão Geriátrica } & \multicolumn{2}{c|}{ LAVTON (AIVD) } & \multicolumn{2}{c|}{ KATZ (AVD) } \\
Resultado & $\mathrm{Fi}$ & Resultado & $\mathrm{Fi}$ & Resultado & $\mathrm{Fi}$ & Resultado & $\mathrm{Fi}$ \\
\hline 13 & 3 & 12 & 01 & 17 & 3 & $\mathrm{~A}$ & 5 \\
12 & 2 & 09 & 01 & 13 & 2 & $\mathrm{C}$ & 2 \\
11 & 5 & 07 & 01 & 112 & 1 & $\mathrm{D}$ & 4 \\
09 & 2 & 06 & 02 & 8 & 1 & $\mathrm{E}$ & 1 \\
07 & 1 & 05 & 01 & 7 & 9 & - & 4 \\
03 & 1 & 04 & 02 & - & - & - & - \\
02 & 1 & 03 & 02 & - & - & - & - \\
NA & 1 & 02 & 01 & - & - & - & - \\
\hline
\end{tabular}

Quadro 1. Distribuição do desempenho dos clientes nos testes de avaliação cognitiva, do humor e da capacidade funcional. Rio de Janeiro, Ambulatório de Neurogeriatria, NAI/UnATI, 2004. 
alimentares do idoso. Importantes cuidados são: consistência da alimentação, suplementação alimentar, postura da alimentação, fracionamento da dieta e ambiente adequado.

A enfermeira tem um papel central nos curativos devemos treinar os cuidadores para realizar o curativo. Este deve ser baseado no cuidado dialógico, sendo que a enfermeira primeiro escuta como os cuidadores realmente realizam o curativo e depois orienta de acordo com as possibilidades de compreensão, aceitação e principalmente com elo de confiança. Se a enfermeira não abre espaço para que ele diga tudo que ele faz com a ferida e se ele não acreditar na forma como a enfermeira orientou a realizar 0 curativo, 0 cuidador não o fará, e poderá passar a seguir conselhos de qualquer pessoa leiga. Todos os cuidadores da pesquisa relatavam ser uma atividade que proporcionava estresse, isso porque além de todos os outros cuidados ainda precisou aprender mais uma habilidade.

Na próxima tabela (2) observamos algo bastante interessante, que não são os cuidados em si o mais difícil, mas sim aprender lidar com os distúrbios de comportamento. Para se entender devemos lembrar que a síndrome demencial acontece num individuo idoso que tinha um papel na família, geralmente o marido, o pai ou o parente de alguém, um indivíduo com história de vida que não tinha tais comportamentos e agora na velhice apresenta dificuldades na comunicação, agressividade, hipersexualidade ou agitação.

Tabela 2 - Distribuição dos cuidados segundo as dificuldades encontradas pelos cuidadores.Rio de Janeiro, Ambulatório de Neurogeriatria, 2004.

\begin{tabular}{cc}
\hline DIFICULDADES NOS CUIDADOS & Fi \\
\hline Comunicação & 4 \\
Comportamento & 4 \\
Agressividade & 4 \\
Supervisionar o idoso nos cuidados & 3 \\
Confiito Familiar & 2 \\
Angustia do Asilamento & 1 \\
Barho & 1 \\
Agitação e Insónia & 1 \\
Preocupações com acidentes & 1 \\
Sem dficuldades & 1 \\
\hline TotalV Relativo & $\mathbf{2 2}$ \\
\hline
\end{tabular}

O item supervisionar o idoso nos cuidados caracteriza a dificuldade do cuidador em se "adaptar" a dependência do idoso ${ }^{(7,8,12)}$. Preocupações com acidentes e sem dificuldades podem caracterizar um posicionamento do cuidador em estresse. A primeira vista pode parecer estranho, mas o indivíduo que não identifica dificuldades pode estar num processo de negação. É importante dar atenção a este item para melhor investigarmos em equipe interdisciplinar o que o individuo que dizer com não ter dificuldades.

É real que o idoso que desenvolve um processo de demência tem $50 \%$ mais de chances de asilamento ${ }^{(13)}$. No evoluir da síndrome mais suporte de cuidados vão sendo necessários (pessoas e dinheiro), se o cuidador não tem este suporte asilamento provavelmente ocorrerá. Verifica-se nas tabelas 1 e 2 que os cuidadores atendem às necessidades de higiene, alimentação e terapêutica medicamentosa dos clientes, mas relatam dificuldades para desenvolver estes cuidados. Tais dificuldades Ihes provocam desgaste físico e emocional, em 100\% dos casos pesquisados.

\section{Problemas de Enfermagem}

Os três principais problemas de enfermagem encontrados nos 16 clientes indicam as três habilidades e competências que a enfermeira deve possuir para trabalhar com idosos com síndrome demencial. Primeiro a Estratégia Inadequada de Resolução de problemas Familiares, fato bastante evidenciado em pesquisas brasileiras onde um único familiar é "escolhido" para cuidar ${ }^{(4,5,12)}$ causando conflitos familiares. Isso acaba por não permitir um manejo adequado no cuidado ao idoso como: o rodízio dos cuidadores e uma distribuição nos gastos financeiros. A enfermeira deve saber atuar na dinâmica familiar tanto em atendimentos individuais quanto em grupo, sempre orientando as melhores estratégias de resolução dos conflitos juntamente com a equipe interdisciplinar, importante salientar que nem sempre o que é bom para o idoso é bom para a família, ou vice-versa, e saber usar do processo de cuidar dialógico é a melhor forma de evitar injustiças.

O Esquecimento requer da enfermeira habilidades e competências no cuidar em reabilitação cognitiva, sejam orientando o familiar de como potencializar a cognição do idoso em domicilio, ou em oficinas de memória, reminiscências, jogos, cuidados pessoais, da palavra, do jornal ou do corpo. Ingesta Hídrica Insuficiente indica que além dos conhecimentos de reabilitação, de dinâmica familiar a enfermeira também desenvolve suas habilidades do cuidado com o corpo biológico, tanto diretamente como em orientações.

Entre os diagnósticos freqüentemente encontrados nos clientes, apresentados na tabela 3 , predominam processo de pensamento alterado, desorientação e agressividade que estão ligados tanto à alteração de comportamento prevista na síndrome demencial|(1-3) ${ }^{(20 m o}$ também, muitas vezes esta relacionada ao manejo inadequado do cuidador com o idoso provocando tais reações ${ }^{(6,9,10,14,15,16)}$.

Tabela 3 - Distribuição dos Diagnósticos de Enfermagem freqüentemente encontrados nos clientes atendidos no Ambulatório de Neurogeriatria. Rio de Janeiro, 2003.

\begin{tabular}{cc}
\hline DIAGNÓSTICO DE ENFERMAGEM & Fi \\
\hline Processo de Pensamento Alterado & 9 \\
Desorientaçäo & 9 \\
Desconforto oral & 9 \\
Agressividade & 8 \\
Pele seca & 8 \\
Incontinència urinária & 7 \\
Emagrecimento & 6 \\
Imobilidade parcial & 5 \\
Dificuldades para dormir & 5 \\
Visäo alterada & 5 \\
Déficit no autocuidado & 5 \\
Diminuição das atividades & 5 \\
Distúrbio no Relacionamento Familiar & 5 \\
\hline
\end{tabular}

O desconforto oral (prótese inadaptada, halitose, higiene oral comprometida), seguido de pele seca, incontinência urinária, emagrecimento e imobilidade parcial e conseqüentemente o déficit do auto cuidado, revelam a dificuldade dos idosos nas atividades de vida diária e a necessidade de cuidados integrais. Constatou-se igual quantitativo na distribuição dos diagnósticos de enfermagem relacionados aos cuidadores dos clientes (vide tabela 4). Este fato nos leva a inferir que a ineficiente comunicação entre cuidador e cliente pode estar condicionada ao despreparo do cuidador para lidar com 0 cliente. Do mesmo modo, a conseqüência esperada desta dificuldade pode ser 0 desgaste do cuidador na execução do cuidar. A Falta de conhecimento do cuidador refere-se também a necessidade de muitas habilidades para o cuidar do idoso com síndrome demencial, pois a cada dia o idoso apresenta com um novo sintoma, considerando a progressão da doença e as flutuações de comportamento do idoso, o cuidador está aprendendo uma nova forma de cuidar diariamente.

Tabela 4- Distribuição dos Diagnósticos de Enfermagem relacionados diretamente com o cuidador dos clientes atendidos no ambulatório de Neurogeriatria. Rio de Janeiro, 2004.

\begin{tabular}{cc}
\hline DIAGNÓSTICO DE ENFERMAGEM & Fi \\
\hline Comunicaçäo Ineficiente entre cuidador e idoso & 6 \\
Desgaste do cuidador & 6 \\
Falta de conhecimento do cuidador & 6 \\
\hline
\end{tabular}


Os resultados evidenciam que para a enfermeira cuidar de um idoso com síndrome demencial deve estar preparada para atuar no binômio idoso-família em três dimensões: a) dimensão biológica- cuidar do corpo físico promovendo conforto e evitando complicações; b) dimensão cognitiva- 0 estudo mostra a necessidade de atuação nesta área- há quatro anos estamos desenvolvendo oficinas para idosos com demência e grupo de familiares e presenciamos a eficácia deste tipo de cuidado. Aqui está a maior barreira a serem vencidos, poucos acreditam nas possibilidades de reabilitação cognitiva no idoso com demência, além de poucos profissionais estarem preparadas para tal cuidado; c) dimensão familiar- 0 cuidado com os cuidadores e os problemas familiares são alvo de muitos estudos e a enfermagem tem um papel pioneiro e importante nesta área, porém o problema continua como demonstrado na pesquisa. É importante avaliar como a enfermagem tem realizado este cuidado, se de forma transmissora ou cuidativa.

\section{CONCLUSÕES}

Podemos afirmar que o estudo conseguiu atingir os três objetivos propostos, demostrando uma relação de variáveis originais em Pesquisa na Enfermagem Brasileira. Os idosos atendidos no ambulatório de Neurogeriatria são na maioria do sexo feminino, na faixa de idade de 70 a 80 anos, a maioria possuem o ensino fundamental completo e ganham um Salário Mínimo, estes residem em casa própria com prevalência dos lares Multigeracionais.

Os testes corroboram com o esperado, de acordo com a literatura ${ }^{(1,2)}$ :
A média de pontuação do MEEM no grupo foi 11; a capacidade funcional encontra-se comprometida primeiramente nas atividades instrumentais como demonstra os resultados da escala de Lawton (AIVD) e posterior comprometimento das atividades de vida diária Katz (AVD); já o teste de EDG não se mostrou aplicável quando se tem um processo demencial instalado.

Quanto aos cuidadores temos a maioria do sexo feminino; com faixa etária predominante de 60-70. Todos os cuidadores realizam cuidados diretos com o indivíduo (preparo da alimentação e Administração dos medicamentos) o que corrobora com a triáde: os testes de avaliação da capacidade funcional; às dificuldades apresentadas; e em seguida com os diagnósticos de enfermagem. As dificuldades apresentadas são em sua maioria relacionadas ao Relacionamento Interpessoal Ineficaz entre INDIVÍDUO-CUIDADOR-FAMÍLIA.

Os Diagnósticos de Enfermagem nos certifica do complexo eixo de cuidados de ENFERMAGEM que o idoso com Síndrome Demencial apresenta: PSICOSSOCIAIS-FAMILIARES-FISIOLOGICOS, exigindo da enfermeira uma estreita habilidade e competência para lidar tanto com os problemas de esquecimento e comportamento quanto aos de higiene, e ao mesmo tempo estar, aberta para ouvir o cuidador.

A ENFERMEIRA é um elemento-chave para um cuidado integral ao indivíduo com Demência, devido à sua habilidade de lidar com a saúde do idoso, cuidador e família, visando sempre à promoção de uma vida mais digna e de qualidade a todos.

\section{REFERÊNCIAS}

1. Machado JCB. Doença de Alzheimer. In: Freitas EV, et al. Tratado de geriatria e gerontologia. Rio de Janeiro (RJ): Guanabara-Koogan; 2002. p. $133-47$

2. Forlenza OV, Caramelli P. Neuropsiquiatria geriátrica. São Paulo (SP): Editora Atheneu; 2000.

3. Busse EW, Blazer DG. Psiquiatria geriátrica. $2^{\mathrm{a}}$ ed. Porto Alegre(RS): Artmed; 1999.

4. Mendes PMT. Cuidadores heróis anônimos do cotidiano. In: Karsch UMS, organizadores. Envelhecimento com dependência: revelando cuidadores. São Paulo (SP): EDUC, 1998.

5. Caldas CP. Envelhecimento com dependência: responsabilidades e demandas da família . Cad Saúde Pública 2003;19(3):733-81.

6. Santana RF. Grupo de Orientação em Cuidados na Demência: relato de experiência de uma enfermeira aluna do curso de psicogeriatria. Textos sobre envelhecimento 2003;5(9).

7. Caldas CP. Cuidando de uma pessoa idosa que vivencia um processo de demência numa perspectiva existencial. In: Guerreiro T, Caldas CP. Memória e demência: (re) conhecimento e cuidado. Rio de Janeiro (RJ): UERJ, UnATI; 2001.

8. Neri AL, organizador. Cuidar de Idosos no contexto da família: questões psicológicas e sociais. Campinas (SP): Editora Alínea; 2002.
9. Savonitti BHR. A. Cuidando do idoso com demência. In: Duarte YAO, Diogo MJD'E. Atendimento domiciliar: um enfoque Gerontológico. São Paulo (SP): Editora Atheneu; 2000. p. 421-38.

10. Rodrigues RAP, Diogo M JDE. Como cuidar dos idosos. Campinas (SP): Papirus; 1996.

11. Araújo LAO, Santana RF, Bachion MM. Mobilidade física prejudicada em idosos: fatores relacionados e características definidoras. Rev Bras Enferm 2002;55(1):19-25.

12. Santos SMA. Idosos, família e cultura: um estudo sobre a construção do papel do cuidador. Campinas (SP): Editora Alínea; 2003.

13. Calkins $E$, Ford $A B$, Karz PR. Psychogeriatric care in the community. Public Health Europe 1992:10.

14. Santos SSC. Enfermagem gerongeriátrica: reflexão à ação cuidativa. São Paulo (SP): Robe Editorial; 2001.

15. Santana RF. Grupo de Orientação em Cuidados na Demência [monografia]. Rio de Janeiro (RJ): Instituto de Psiquiatria da Universidade do Brasil/UFRJ; 2003.

16. Mailloux-Poirer M. Comunicar. In: Berger LM, Mailloux-Poirer M. Pessoas idosas: uma abordagem global. Lisboa (PT): Editora Lusodidacta; 1995 . p. 475-502. 